\title{
A Survey of e-Commerce Recommender Systems
}

\author{
Farida Karimova, $P h D$
}

School of Management

Huazhong University of Science and Technology, China

\section{doi: 10.19044/esj.2016.v12n34p75 URL:http://dx.doi.org/10.19044/esj.2016.v12n34p75}

\begin{abstract}
Due to their powerful personalization and efficiency features, recommendation systems are being used extensively in many online environments. Recommender systems provide great opportunities to businesses, therefore research on developing new recommender system techniques and methods have been receiving increasing attention. This paper reviews recent developments in recommender systems in the domain of ecommerce. The main purpose of the paper is to summarize and compare the latest improvements of e-commerce recommender systems from the perspective of e-vendors. By examining the recent publications in the field, our research provides thorough analysis of current advancements and attempts to identify the existing issues in recommender systems. Final outcomes give practitioners and researchers the necessary insights and directions on recommender systems.
\end{abstract}

Keywords: E-Commerce, Recommender Systems, Survey

\section{Introduction}

Over the last decade there has been a lot of focus and tremendous interest towards recommender systems from e-commerce providers. Recommender systems (RSs) can be defined as information filtering system that predict users' preferences and recommend potentially interesting items to these users. (Adomavicius \& Tuzhilin, 2005)

RS is a comparatively new area in information systems research. It emerged as an independent research in the mid-1990s. Since then, different theoretical methods and empirical approaches have been proposed by researchers. (Li \& Karahanna, 2015) There are several types of recommendation techniques, including content-based (CB), collaborative filtering-based (CF) and hybrid approaches. Each recommendation approach has its own limitations. For example, CB has overspecialization problem, while CF has sparsity and cold start problems. 
In recent years, the focus of research has been on solving major drawbacks of existing RSs. The main purpose of this paper is to summarize and compare the latest improvements of e-commerce RSs from the perspective of e-commerce providers. Our research provides thorough analysis of current advancements and attempts to identify the existing issues in RSs by examining the recent publications in the field and provide practitioners and researchers with insights and future directions on RSs.

The major contributions of this paper can be summarized as follows:

1) This paper provides a review and interpretation of RS research in e-commerce domain with the intension of analyzing important issues for further research. Moreover, we provide a detailed summary of e-commerce RSs highlighting the e-commerce providers' perspective.

2) Existing RS surveys mainly focus on either technical aspect of RSs, or for all application domains. This paper surveyed the RS developed especially for e-commerce domain, which complements the existing gap in the RS surveys and provides useful guide for e-vendors and;

3) Drawing upon the literature review and analysis of RS approaches, we discuss several important research issues surrounding the RSs in ecommerce.

4) Finally, our review identifies a number of opportunities for future research on e-commerce RSs.

The remainder of the paper is organized as follows. Section 1 reviews prior literature, research methodology is presented in Section 2. E-commerce RS functions and algorithms are discussed in Section 3. Section 4 is devoted to analyze e-commerce RS from the perspective of e-vendors. Section 5 concludes with a summary.

\section{Related Work}

Several papers on RSs surveys have been published in the last decade in order to analyze major problems of traditional and non-traditional RSs.

Li \& Karahanna (2015) provide a comprehensive research on ecommerce RSs addressing three major areas: understanding consumers, how recommendations work and the impacts of RSs. More akin to the methodology used in this paper, Lu et al. (2015) review the latest application developments of RSs in several application domains such as e-government, e-business and e-commerce through recommendation methods, software, and application platforms. While, Adomavicius and Tuzhilin (2005) reviewed the RSs, such as content-based, collaborative filtering-based and hybrid approaches; and discussed their limitations and possible solutions to enhance recommendation performance. 
This review is distinguishable from these papers in that the existing works mainly focus on RS methods and approaches, while none of these survey papers provide the analysis of RSs from e-vendors' perspective.

\section{Research Methodology}

In this research, two types of articles that were published in the last three years were reviewed and classified: 1) Articles on RS techniques, specifically related methods and approaches; 2) Articles on RSs designed particularly for e-commerce domain.

In order to select high impact and innovative articles, the selection process was based on the following criteria: 1) published within the last three years (i.e. from 2014-2016); 2) published in high impact factor journals and conference proceedings.

The research and selection of these articles were performed as follows:

A. The following journal databases were used in order to select research papers on RSs for our study: ACM Digital Library, IEEE Xplore, Science Direct and SpringerLink.

B. Therefore, the search was implemented based on related keywords of RSs in e-commerce (such as, recommender system, ecommerce recommender system), while holding the following two criteria:

1) The articles must propose a novel recommender approach which addresses current limitations; 2) The articles must propose a novel recommender technique designed for e-commerce.

C. Based on the keywords related to e-commerce domain, these papers were divided by RS methods such as collaborative filtering-based, content-based, hybrid approaches and social-network based techniques. For each RS method relevant research papers were examined and chosen.

Finally, we selected 60 papers on e-commerce recommender systems for our research.

\section{A Comprehensive Overview: E-commerce Recommender Systems}

Large e-vendors such as Amazon.com, eBay.com and Taobao.com are the best examples of massive implementers of recommender systems. The products are usually recommended based on popularity, customer demographics and analysis of the customer's past purchase behaviors. (Schafer et al., 1999) Purchased product rating is a common function in eshops. For example, in Amazon.com, feedbacks for purchased items are provided by giving a rating between 1 and 5 . These ratings data can be used to make recommendations. (Lu et al., 2015) 


\section{E-commerce Recommender System functions}

RSs have big impact on users and on e-commerce providers. However, e-vendors and users' incentives to use RS may vary. (Ricci et al., 2011) For example, one of the reasons e-vendors introduce ecommerce RS is to increase the number of items sold. Whereas, the RSs alleviate information overload problem and help users find a better product by providing personalized services. (Adomavicius \& Tuzhilin, 2005)

We define the motivations behind introducing RS to customers by service providers as follows (Ricci et al., 2011):

1) Acceptance helps to increase the conversion rate. The primary goal of implementing a RS in e-commerce is to increase the conversion rate i.e. being able to sell more recommended items compared to those usually sold without any method of RS.

2) Accuracy yields increase in the user satisfaction. Accuracy is one of the major functions of RS. If a user finds recommended items relevant and interesting, the probability of the recommended items' acceptance will be high.

3) RS increases user loyalty by providing personalized services. RS helps to gain user loyalty, which in turn increases the conversion rate. Subsequently, loyal users' preferences will be easier to predict, as a result, the recommended items accuracy will increase. (Wei et al., 2007) Moreover, the system should also be able to recognize new users and accurately provide services in order to gain new user's loyalty.

4) RS helps to sell more diverse items. Recommending diverse, yet relevant items is considered to be one of the premises that the user enjoys using the RS. Hence, diversity increases user loyalty and the number of diverse items sold.

\section{Recommender System algorithms}

In order to analyze e-commerce RS developments, we must first review the main state-of-the-art recommendation techniques such as contentbased, collaborative filtering-based, and hybrid methods and a nontraditional method, such as social network-based approach.

\section{Content-based filtering recommendation technique}

Content-based (CB) filtering recommendation technique recommends items similar to the items the user purchased in the past. (Adomavicius \& Tuzhilin, 2005) The bottleneck of the CB recommender techniques is to analyze the content of the items preferred by a particular user to determine the user's preferences. Usually, two techniques are used in order to generate recommendations. 1) Using traditional information retrieval methods, such as cosine similarity measure, Tf-Idf, LDA. 2) Using advanced machine 
learning methods, such as Naïve Bayes, vector support machines, decision trees.

Content-based filtering recommendations have three major limitations ( $\mathrm{Li} \&$ Karahanna, 2015): overspecialization, when a recommender is not able to recommend unexpected, yet suitable items; eliciting user feedback, where the recommendation quality can be improved only with user's more historical data. For example, if a user purchases or views products or provides feedbacks to products.

\section{Collaborative filtering based recommendation technique}

Collaborative filtering (CF) based recommender systems recommend an item for a particular user based on the items previously preferred by other users. (Adomavicius \& Tuzhilin, 2005) There are two types of CF technique:

1) Memory-based algorithms (such as, item-based and user-based CF approaches) - recommendations are generated based on the preferences of the nearest neighbors. (Al-shamri, 2014). In the item-based CF approach, a user receives recommendations that are similar to those he rated in the past. While in the user-based CF approach, recommended items are based on the similar users. The similarity between users or items can be calculated by Pearson correlation coefficient, cosine similarity measures. (Al-shamri, 2014). Empirical results prove that, item-based CF algorithm outperforms user-based approach. (Alqadah et al., 2015)

2) Model-based algorithms such as Matrix Factorization algorithms such as SVD, tensor factorization, Restricted Boltzmann machines, Bayesian networks make recommendations by exploring latent features of user ratings or by building a model in order to predict the most preferable item which a user may wish to purchase. (Alqadah et al., 2015)

CF based techniques are widely used in building e-commerce RSs. (Zhao et al., 2015) However, CF has problems such as cold start, scalability and data sparsity. (Guo et al., 2014) Data sparsity refers to the condition of low percent of rated items over the whole number of items. Cold start problem means the condition where there are new users or item with little historical behavior.

\section{Hybrid recommendation techniques}

Each RS techniques has its own limitations, it is difficult to solve all issues with one method. (Zhao et al., 2015) Therefore, Hybrid recommendation techniques emerged. Hybrid recommendation techniques combine two or more recommendation methods, such as $\mathrm{CB}$ and $\mathrm{CF}$ based techniques in order to avoid certain limitations such as cold-start problem, while improving RS performance. (Adomavicius \& Tuzhilin, 2005) There are different ways to create a new hybrid RS: weighted, switching, mixed, 
feature combination, cascade, feature augmentation and meta-level. Hybrid filtering is usually based on probabilistic methods such as neural networks, Bayesian networks, clustering, latent features (e.g. SVD), genetic algorithms. (Beladev et al., 2016)

Although Hybrid approaches are solution for many existing RS techniques, (Quieroz et al., 2016) more information and effort is required to implements this technique.

\section{Social network-based recommendation techniques}

Social network-based (SN) recommender techniques utilizes information in social networks, such as user preferences, influence from social friends in order to improve recommendation accuracy and to overcome major challenges, such as cold-start and data sparsity problems. (Chen et al., 2013) This technique is based on the fact that social friends share certain interests in common. (Yu et al., 2016) SN creates communitybased recommendations by measuring users' rating similarities. (Yu et al., 2016) SN recommender systems are based on probabilistic methods such as Bayesian networks and neural networks. (Li et al., 2015)

\section{The analysis of existing e-commerce recommender systems from the perspective of e-vendors}

For several years great effort has been devoted to the study of improving business functions of RSs. Kitayama et al. (2015) proposed a new e-commerce RS based on a user's viewpoint for specialty shops that sell unique items. They use the item classification method in order to determine the shop's degree of specialty. But the method's main limitation is that it is unknown whether specialty items can be found in big general e-stores. Peska et al. (2016), on the other hand, presented a novel approach that tries to enhance RSs in small and medium e-commerce providers. Specifically, they focus on the low consumption rate and user loyalty problem by learning from implicit user feedback. Beladev et al. (2016) proposed a novel model of bundle recommendations using CF, demand functions and price modeling. It is specialized to maximize the expected revenue of recommended products by finding pairs of items which helps to maximize both the probability of product purchase and the revenue. Also, Hernando et al. (2016) presented a new approach based on CF method that predicts users' tastes.

Cold-start and long tail problems in e-commerce RSs still remain to be a salient part of research. Valcarce et al. (2016), Hu et al. (2014) propose a new approach that can be implemented to mitigate long tail products while maximizing recommendation accuracy. Yu et al. (2016) proposed a new approach by combining users' sentiments in review texts in order to overcome cold-start problem. Moreover, Yu et al. (2016) presented a 
different approach in order to overcome item cold-start problem by utilizing MF by incorporating item-attribute information. Recently, Hoang et al. (2015), Pereira and Raul (2015) have proposed a novel hybrid method for the new user cold start problem and demonstrate this method outperforms traditional methods by recommendation accuracy.

In the last three years, accuracy improvement and low computational complexity of recommendation algorithms have attracted much attention from researchers. Specifically, Hu et al. (2015), Koohi and Kiani (2016), Moradi and Ahmadian (2015), Nguyen and Huynh (2016), Polatidis and Georgiadis (2016), Queiroz et al. (2016), Wang et al. (2016), Xu et al. (2014) propose new methods for e-commerce providers. They demonstrate that these approaches outperform the state-of-the-art techniques in terms of recommendation accuracy and scalability. Moreover, lowering complexity in computation plays a key part in the improvement in the performance of RSs. Javari et al. (2014), Zhao et al. (2015) propose new techniques to decrease computational complexity of RSs, while Kaminskas et al. (2016) apply a hybrid approach which combines association rules and text-based similarity by demonstrating the effectiveness of this approach.

Liao et al. (2016), Rana and Kumar (2014), Zhang et al. (2016) presented innovative approaches which apply clustering algorithm using CF in order to improve efficiency and lower computation complexity without compromising the recommendation quality.

Table 1. Summary of E-Commerce Recommender Systems in Prior Studies

\begin{tabular}{|c|c|c|c|}
\hline Papers & Information Source & Examined Problems & $\begin{array}{c}\text { Implemented RS } \\
\text { technique }\end{array}$ \\
\hline $\begin{array}{c}\text { Totally: } 2 \text { papers } \\
\text { Peska, \& Vojtas } \\
\text { (2016), Kitayama et } \\
\text { al. (2015). }\end{array}$ & $\begin{array}{c}\text { Implicit user } \\
\text { feedback, category } \\
\text { information. }\end{array}$ & $\begin{array}{l}\text { Easy discovering specialty } \\
\text { items. Also unique } \\
\text { challenges, such as scarcity } \\
\text { of explicit feedback, user } \\
\text { loyalty problem, low } \\
\text { consumption rate. }\end{array}$ & $\begin{array}{l}\text { Content-based } \\
\text { filtering }\end{array}$ \\
\hline $\begin{array}{l}\text { Totally: } 24 \text { papers } \\
\text { Alqadah et al. (2015), } \\
\text { Al-shamri, M. Y. H. } \\
\text { (2014), Beladev et al. } \\
\text { (2016), Guo et al. } \\
\text { (2014), Hernando et } \\
\text { al. (2016), Hu et al. } \\
\text { (2015), Huang et al. } \\
\text { (2016), Javari et al. } \\
\text { (2014), Köhler, S. } \\
\text { (2016), Koohi, H., \& } \\
\text { Kiani, K. (2016), } \\
\text { Liao, C., \& Lee, S. } \\
\text { (2016), Liu et al. } \\
\text { (2014), Nguyen, V., } \\
\text { \& Huynh, V. (2016), } \\
\text { Polatidis, N., \& }\end{array}$ & $\begin{array}{c}\text { Implicit feedback } \\
\text { data such as review } \\
\text { texts, user ratings, } \\
\text { number of } \\
\text { purchases, item } \\
\text { price, pairwise } \\
\text { interactions, item- } \\
\text { attribute } \\
\text { information. }\end{array}$ & $\begin{array}{c}\text { Data sparsity, limited } \\
\text { scalability problem, user and } \\
\text { item cold start problems, } \\
\text { long-tail problem. } \\
\text { Improvements in } \\
\text { recommendation diversity. } \\
\text { Accuracy in prediction and } \\
\text { recommendations, advances } \\
\text { in user similarity measures, } \\
\text { advances in predicting the } \\
\text { user tastes, personalized } \\
\text { price recommendations, } \\
\text { maximizing the expected } \\
\text { revenue, increase in number } \\
\text { of purchases. Also, } \\
\text { improvements in } \\
\text { performance i.e. decrease in }\end{array}$ & $\begin{array}{l}\text { Collaborative } \\
\text { filtering-based }\end{array}$ \\
\hline
\end{tabular}




\begin{tabular}{|c|c|c|c|}
\hline $\begin{array}{l}\text { Georgiadis, C. K. } \\
\text { (2016), Valcarce } \\
\text { (2016), Wang et al. } \\
\text { (2016), Xu et al. } \\
\text { (2014), Yera et al. } \\
\text { (2015), Yu et } \\
\text { al.(2015), Yu et al. } \\
\text { (2016), Zhang et al. } \\
\text { (2016), Zhang, Z., } \\
\text { Zheng, X., \& Dajun, } \\
\text { D. (2016), Zhao et al. } \\
\text { (2015), Zhou et al. } \\
\text { (2016). }\end{array}$ & & $\begin{array}{l}\text { computational complexity, } \\
\text { improvements in the } \\
\text { technique efficiency, } \\
\text { increase customer loyalty }\end{array}$ & \\
\hline $\begin{array}{c}\text { Totally: } 9 \text { papers } \\
\text { Hoang, L. (2015), Hu } \\
\text { et al. (2014), Hussein } \\
\text { et al. (2014), Javari } \\
\text { (2015), Kaminskas, } \\
\text { M., Bridge, D., } \\
\text { Foping, F., \& Roche, } \\
\text { D. (2016), Luiz, A., } \\
\text { Pereira, V., \& Raul, } \\
\text { E. (2015), Nilashi, } \\
\text { M., Ibrahim, O., \& } \\
\text { Ithnin, N. (2014), } \\
\text { Qiu, L., Gao, S., } \\
\text { Cheng, W., \& Guo, J. } \\
\text { (2016), Studies, I. J. } \\
\text { H., Parra, D., \& } \\
\text { Brusilovsky, P. } \\
\text { (2015). }\end{array}$ & $\begin{array}{l}\text { Demographic data, } \\
\text { rating data, items' } \\
\text { content (textual } \\
\text { data), purchase } \\
\text { history, shoppers' } \\
\text { browsing history, } \\
\text { review texts. }\end{array}$ & $\begin{array}{l}\text { User cold start problem, user } \\
\text { preferences, long-tail } \\
\text { problem, style preferences, } \\
\text { Accuracy problem, Low } \\
\text { computational complexity, } \\
\text { Novelty problem, User } \\
\text { acceptance. }\end{array}$ & Hybrid approaches \\
\hline $\begin{array}{c}\text { Totally: } 2 \text { papers } \\
\text { Li, Y., Chou, C., \& } \\
\text { Lin, L. (2015), Zhao, } \\
\text { W. X., Li, S., He, Y., } \\
\text { \& Chang, E. Y. } \\
\text { (2016). }\end{array}$ & $\begin{array}{l}\text { Demographic data, } \\
\text { product } \\
\text { characteristics. }\end{array}$ & $\begin{array}{l}\text { Increase in willingness to } \\
\text { purchase, user preferences, } \\
\text { item cold start problem. }\end{array}$ & Social Network-based \\
\hline
\end{tabular}

For e-commerce domain, the recommendation techniques are summarized and presented in Table 1. From the summary of RSs, the following important findings can be extracted:

1) Results of survey analysis show that, the traditional RS techniques, such as CF and Hybrid play a dominant part in e-commerce RS. Research on CF based RSs are used more than other approaches; Moreover, researchers have been concentrated on to overcome the limitations based on CF algorithms, such as decreasing computational time, improving recommendation accuracy. As a result, CF covers the functions such as, accuracy, acceptance, selling more diverse items and improving existing users' loyalty. Although user cold start problem is still under research.

2) Several papers suggest novel algorithms to mitigate dimensionality problem. In order to generate recommendations, it is computationally 
inefficient to consider every item in a huge e-shop. Experimental results show that the efficiency of RS is improved without compromising the recommendation accuracy.

3) For the last three years, great effort has been devoted to the study of minimizing computational complexity to maximize RS efficiency using CF approach;

4) Based on the research, social-network RSs also played important role in recent approach developments. Increase in willingness to purchase, item cold start problem, accuracy in user preferences are main topics addressed by researchers using SN based technique;

5) The aim of the RS for service providers is to achieve more accuracy. So finding best similar users and achieving higher recommendation accuracy is a major challenge in RS. Based on the analysis, a fruitful research was done on improving similarity measure and clustering algorithms which are major disadvantages of existing traditional techniques. In order to overcome these shortages innovative methods were introduced. Compared to traditional clustering algorithms, new clustering algorithms can provide much accurate predictions with low computational cost.

Although productive research has been done on creating new recommendation methods, there are still major drawbacks to be considered:

1) Although there are a few studies concentrated on user and item cold start problem, further study of this issue using advanced methods is still required. One possible way is to use social media information when a user has only limited historical behavior. Little is known about how the social media information may help to overcome cold start problem. Besides, more detailed structural information on the users and items might shed light on mitigating cold start problem.

2) Data sparsity and limited scalability. Constructing a more effective non-linear mapping function to reduce the amount of data may help to overcome this limitation. (Zhao et al., 2015)

3) Diversity and serendipity of recommendations. It would be more interesting to study how the balance between diversity and prediction accuracy can improve the recommendation performance. Exploring other RS algorithms such as item popularity, social network could be a possible solution.

4) Recent research indicates the potential importance of examining the impact of the dynamic user behavior and ratings behavior of users. User preferences are dynamic and changes over time. Many existing models are unable to capture the latest changes in user preferences over time.

5) Social-media based RS and other RS techniques. Further investigation on more sophisticated approaches such as context-aware recommender systems, group recommendation systems should be conducted. 
6) Further research is necessary to better understand various factors that affect recommendation accuracy. For example, price. As price plays important factor in purchasing, little is known whether adjusting the pricing strategy for a RS will help to achieve an accurate performance.

\section{Conclusion}

In order to provide directions for further research and studies, the paper reviewed more than 60 RS methods developed specifically for ecommerce domain to analyze the current developments and issues in ecommerce RS. As result, it is found that the traditional RS techniques, such as CF and Hybrid play a dominant part in e-commerce RS. Moreover, the observations show that researchers have been concentrating on to overcome the limitations based on CF algorithms, such as decreasing computational complexity, improving recommendation accuracy. Therefore, CF covers the functions such as, accuracy, acceptance, selling more diverse items and improving existing users' loyalty. Although productive research has been conducted on attempts to create personalized RS with high accuracy, there are still major drawbacks to be considered. Hence, our review concludes that continuing research on the RS appears fully justified because several issues remain to be addressed.

\section{References:}

1. Liu, C., \& Wu, X. (2016). Large-scale recommender system with compact latent factor model, 64, 467-475. doi:10.1016/j.eswa.2016.08.009

2. Adomavicius, G., \& Tuzhilin, A. (2005). Toward the next generation of recommender systems: A survey of the state-of-the-art and possible extensions. IEEE Transactions on Knowledge and Data Engineering, 17(6), 734-749. doi:10.1109/TKDE.2005.99

3. Schafer, J. Ben, Joseph Konstan, and John Riedl. "Recommender systems in e-commerce." Proceedings of the 1st ACM conference on Electronic commerce. ACM, 1999.

4. Peska, L., \& Vojtas, P. (2016). Using Implicit Preference Relations to Improve Recommender Systems. Journal on Data Semantics. doi:10.1007/s13740-016-0061-8

5. Kitayama, D., Zaizen, M., Sumiya, K. (2015). An E-commerce Recommender System Using Measures of Specialty Shops, 369-383. doi:10.1007/978-94-017-9588-3

6. Alqadah, F., Reddy, C. K., Hu, J., \& Alqadah, H. F. (2015). Biclustering neighborhood-based collaborative filtering method for top- $\mathrm{n}$ recommender systems. Knowledge and Information Systems, 475-491. doi:10.1007/s10115-014-0771-X 
7. Al-shamri, M. Y. H. (2014). Expert Systems with Applications Power coefficient as a similarity measure for memory-based collaborative recommender systems. EXPERT SYSTEMS WITH APPLICATIONS, 41(13), 5680-5688. doi:10.1016/j.eswa.2014.03.025

8. Beladev, M., Rokach, L., \& Shapira, B. (2016). Recommender systems for product bundling R. Knowledge-Based Systems, 111, 193-206. doi:10.1016/j.knosys.2016.08.013

9. Guo, G., Zhang, J., \& Thalmann, D. (2014). Knowledge-Based Systems Merging trust in collaborative filtering to alleviate data sparsity and cold start. Knowledge-Based Systems, 57, 57-68. doi:10.1016/j.knosys.2013.12.007

10. Hernando, A., Bobadilla, J., \& Ortega, F. (2016). Knowledge-Based Systems A non negative matrix factorization for collaborative filtering recommender systems based on a Bayesian probabilistic model, 97, 188-202. doi:10.1016/j.knosys.2015.12.018

11. Hoang, L. (2016). Dealing with the new user cold-start problem in recommender systems : A comparative review. Information Systems, 58, 87-104. doi:10.1016/j.is.2014.10.001

12. Hu, L., Lin, K., Mehedi, M., Alamri, A., \& Alelaiwi, A. (2015). CFSF : On Cloud-Based Recommendation for Large-Scale Ecommerce, 380-390. doi:10.1007/s11036-014-0560-5

13. Huang, T. C., Chen, Y., \& Chen, M. (2016). A novel recommendation model with Google similarity. Decision Support Systems, 89, 17-27. doi:10.1016/j.dss.2016.06.005

14. Javari, A., Gharibshah, J., \& Jalili, M. (2014). Recommender systems based on collaborative filtering and resource allocation. doi:10.1007/s13278-014-0234-0

15. Köhler, S. (2016). The impact of consumer preferences on the accuracy of collaborative filtering recommender systems. Electronic Markets. doi:10.1007/s12525-016-0232-3

16. Koohi, H., \& Kiani, K. (2016). User based Collaborative Filtering using fuzzy C-means. Measurement, 91, 134-139. doi:10.1016/j.measurement.2016.05.058

17. Liao, C., \& Lee, S. (2016). Electronic Commerce Research and Applications A clustering based approach to improving the efficiency of collaborative filtering recommendation. Electronic Commerce Research and Applications, 18, 1-9. doi:10.1016/j.elerap.2016.05.001

18. Liu, H., Hu, Z., Mian, A., Tian, H., \& Zhu, X. (2014). KnowledgeBased Systems filtering q. Knowledge-Based Systems, 56, 156-166. doi:10.1016/j.knosys.2013.11.006 
19. Nguyen, V., \& Huynh, V. (2016). International Journal of Approximate Reasoning Two-probabilities focused combination in recommender. International Journal of Approximate Reasoning, 80, 225-238. doi:10.1016/j.ijar.2016.09.005

20. Polatidis, N., \& Georgiadis, C. K. (2016). A multi-level collaborative filtering method that improves recommendations, 48, 100-110. doi:10.1016/j.eswa.2015.11.023

21. Rana, C., \& Kumar, S. (2014). An evolutionary clustering algorithm based on temporal features for dynamic recommender systems. Swarm and Evolutionary Computation, 14, 21-30. doi:10.1016/j.swevo.2013.08.003

22. Ranjbar, M., Moradi, P., Azami, M., \& Jalili, M. (2015). Engineering Applications of Arti fi cial Intelligence An imputation-based matrix factorization method for improving accuracy of collaborative fi ltering systems. Engineering Applications of Artificial Intelligence, 46, 58-66. doi:10.1016/j.engappai.2015.08.010

23. Valcarce, D., Parapar, J., \& Barreiro, Á. (2016). Knowle dge-Base d Systems Item-based relevance modelling of recommendations for getting rid of long tail products, 103, 41-51. doi:10.1016/j.knosys.2016.03.021

24. Wang, N., Zhang, Q., Yang, L., \& Chen, M. (2016). A Novel Ecommerce Recommendation Algorithm based on Neural Network and Collaborative Analysis, 138(Isi), 99-104.

25. Xu, R., Wang, S., Zheng, X., \& Chen, Y. (2014). The Journal of Systems and Software Distributed collaborative filtering with singular ratings for large scale recommendation. The Journal of Systems \& Software, 95, 231-241. doi:10.1016/j.jss.2014.04.045

26. Yera, R., Caballero, Y., \& Martínez, L. (2015). Knowledge-Based Systems Correcting noisy ratings in collaborative recommender systems. Knowledge-Based Systems, 76, 96-108. doi:10.1016/j.knosys.2014.12.011

27. Yu, D., Mu, Y., \& Jin, Y. (2016). Rating prediction using review texts with underlying sentiments. Information Processing Letters, 117, 10-18. doi:10.1016/j.ipl.2016.08.002

28. Yu, L., Liu, C., \& Zhang, Z. (2015). Knowledge-Based Systems Multi-linear interactive matrix factorization, 85, 307-315. doi:10.1016/j.knosys.2015.05.016

29. Yu, Y., Wang, C., Wang, H., \& Gao, Y. (2016). Attributes coupling based matrix factorization for item recommendation. Applied Intelligence. doi:10.1007/s10489-016-0841-8 
30. Zhang, J., Lin, Y., Lin, M., \& Liu, J. (2016). An effective collaborative filtering algorithm based on user preference clustering. Applied Intelligence, 230-240. doi:10.1007/s10489-015-0756-9

31. Zhang, Z., Zheng, X., \& Dajun, D. (2016). Knowle dge-Base d Systems A framework for diversifying recommendation lists by user interest expansion, 105, 83-95. doi:10.1016/j.knosys.2016.05.010

32. Zhao, Y.-S., Liu, Y.-P., \& Zeng, Q.-A. (2015). A weight-based item recommendation approach for electronic commerce systems. Electronic Commerce Research. doi:10.1007/s10660-015-9188-1

33. Zhou, X., \& Wu, S. (2016). Knowle dge-Base d Systems Rating LDA model for collaborative filtering. Knowledge-Based Systems, 110, 135-143. doi:10.1016/j.knosys.2016.07.020

34. Hoang, L. (2015). Engineering Applications of Arti fi cial Intelligence HU-FCF $\mathrm{p} \mathrm{p}$ : A novel hybrid method for the new user cold-start problem in recommender systems. Engineering Applications of Artificial Intelligence, 41, 207-222. doi:10.1016/j.engappai.2015.02.003

35. Hu, D. J., Hall, R., \& Attenberg, J. (2014). Style in the long tail: Discovering unique interests with latent variable models in large scale social e-commerce. Sigkdd, 1640-1649. doi:10.1145/2623330.2623338

36. Hussein, T., Linder, T., \& Gaulke, W. (2014). Hybreed : A software framework for developing context-aware hybrid recommender systems, 121-174. doi:10.1007/s11257-012-9134-z

37. Javari, A., \& Jalili, M. (2015). A probabilistic model to resolve diversity - accuracy. Knowledge and Information Systems, 609-627. doi:10.1007/s10115-014-0779-2

38. Kaminskas, M., Bridge, D., Foping, F., \& Roche, D. (2016). ProductSeeded and Basket-Seeded Recommendations for Small-Scale Retailers. Journal on Data Semantics. doi:10.1007/s13740-016-00583

39. Luiz, A., Pereira, V., \& Raul, E. (2015). Knowledge-Based Systems Simultaneous co-clustering and learning to address the cold start problem in recommender systems. Knowledge-Based Systems, 82, 11-19. doi:10.1016/j.knosys.2015.02.016

40. Nilashi, M., Ibrahim, O., \& Ithnin, N. (2014). Expert Systems with Applications Hybrid recommendation approaches for multi-criteria collaborative filtering. EXPERT SYSTEMS WITH APPLICATIONS, 41(8), 3879-3900. doi:10.1016/j.eswa.2013.12.023

41. Qiu, L., Gao, S., Cheng, W., \& Guo, J. (2016). Knowle dge-Base d Systems Aspect-based latent factor model by integrating ratings and 
reviews for recommender system. Knowledge-Based Systems, 110, 233-243. doi:10.1016/j.knosys.2016.07.033

42. Studies, I. J. H., Parra, D., \& Brusilovsky, P. (2015). Usercontrollable personalization: A case study with SetFusion \$. Journal of

43. man Computer Studies, 78, 43-67. doi:10.1016/j.ijhcs.2015.01.007

44. Li, Y., Chou, C., \& Lin, L. (2015). A social recommender mechanism for location-based group commerce. Information Sciences, 274(2014), 125-142. doi:10.1016/j.ins.2014.02.079

45. Zhao, W. X., Li, S., He, Y., \& Chang, E. Y. (2016). Connecting Social Media to E-Commerce : Cold-Start Product Recommendation Using Microblogging Information, 28(5), 1147-1159.

46. Moradi, P., \& Ahmadian, S. (2015). Expert Systems with Applications A reliability-based recommendation method to improve trust-aware recommender systems. Expert Systems With Applications, 42(21), 7386-7398. doi:10.1016/j.eswa.2015.05.027

47. Queiroz, E., Camilo-junior, C. G., Pascoal, L. M. L., \& Rosa, T. C. (2016). An evolutionary approach for combining results of recommender systems techniques based on collaborative filtering. Expert Systems With Applications, 53, 204-218. doi:10.1016/j.eswa.2015.12.050

48. Li, S. S., \& Karahanna, E. (n.d.). Journal of the Association for Information Systems Online Recommendation Systems in a B2C ECommerce Context: A Review and Future Directions Online Recommendation Systems in a B2C E-commerce Context : A Review and Future Directions, 16(2), 72-107.

49. Lu, J., Wu, D., Mao, M., Wang, W., \& Zhang, G. (2015). Recommender system application developments : A survey. Decision Support Systems, 74, 12-32. doi:10.1016/j.dss.2015.03.008

50. Ricci F., Rokach L., Shapira B., Kantor R.B. (2011) Recommender Systems Handbook Springer New York Dordrecht Heidelberg London ISBN 978-0-387-85819-7

51. Chen, S., Owusu, S., \& Zhou, L. (2013). Social Network Based Recommendation Systems : A Short Survey. doi:10.1109/SocialCom.2013.134

52. B, H. Z., \& Li, J. (2014). Mining Intelligence and Knowledge Exploration, 8891, 504-514. doi:10.1007/978-3-319-13817-6

53. Viriato de Melo, E., Nogueira, E. A., \& Guliato, D. (2015). ContentBased Filtering Enhanced by Human Visual Attention Applied to Clothing Recommendation. 2015 IEEE 27th International Conference on Tools with Artificial Intelligence (ICTAI), 644-651. doi:10.1109/ICTAI.2015.98 
54. Wang, L. C., Zeng, X. Y., Koehl, L., \& Chen, Y. (2015). Intelligent fashion recommender system: Fuzzy logic in personalized garment design. IEEE Transactions on Human-Machine Systems, 45(1), 95109. doi:10.1109/THMS.2014.2364398

55. Yu, F., Zeng, A., Gillard, S., \& Medo, M. (2016). Network-based recommendation algorithms: A review. Physica A, 452, 192-208. doi:10.1016/j.physa.2016.02.021

56. Qian, F., Zhao, S., Tang, J., \& Zhang, Y. (2016). SoRS : Social recommendation using global rating reputation and local rating similarity. Physica A, 461, 61-72. doi:10.1016/j.physa.2016.05.025

57. Wei, K., Huang, J., \& Fu, S. (2007). A Survey of E-Commerce Recommender Systems, (2006).

58. Bok, K., Lim, J., Yang, H., \& Yoo, J. (2016). Neurocomputing Social group recommendation based on dynamic pro fi les and collaborative fi ltering, 209, 3-13. doi:10.1016/j.neucom.2015.11.120

59. Hu, X., Huang, Q., Zhong, X., Davison, R. M., \& Zhao, D. (2016). International Journal of Information Management The influence of peer characteristics and technical features of a social shopping website on a consumer's purchase intention, 36, 1218-1230. doi:10.1016/j.ijinfomgt.2016.08.005

60. Ortega, F., Hernando, A., Bobadilla, J., \& Hyung, J. (2016). Recommending items to group of users using Matrix Factorization based Collaborative Filtering, 345, 313-324. doi:10.1016/j.ins.2016.01.083

61. Boratto, L., Carta, S., \& Fenu, G. (2016). Discovery and representation of the preferences of automatically detected groups : Exploiting the link between group modeling and clustering. Future Generation Computer Systems, 64, 165-174. doi:10.1016/j.future.2015.10.007 